University of Nebraska - Lincoln

DigitalCommons@University of Nebraska - Lincoln

$1-1996$

\title{
Energy and Angular Distributions of Electrons from Ion Impact on Atomic and Molecular Hydrogen. IV. 28-114-keV He$++\mathrm{H}$ Collisions
}

\author{
Y.-Y. Hsu \\ University of Nebraska - Lincoln \\ M. W. Gealy \\ University of Nebraska - Lincoln \\ G. W. Kerby III \\ University of Nebraska - Lincoln \\ M. Eugene Rudd \\ University of Nebraska - Lincoln, erudd@unl.edu \\ D. R. Schultz \\ Physics Division, Oak Ridge National Laboratory, Oak Ridge, Tennessee \\ See next page for additional authors \\ Follow this and additional works at: https://digitalcommons.unl.edu/physicsrudd \\ Part of the Physics Commons
}

Hsu, Y.-Y.; Gealy, M. W.; Kerby III, G. W.; Rudd, M. Eugene; Schultz, D. R.; and Reinhold, C. O., "Energy and Angular Distributions of Electrons from Ion Impact on Atomic and Molecular Hydrogen. IV. 28-114-keV $\mathrm{He}^{+}+\mathrm{H}$ Collisions" (1996). M. Eugene Rudd Publications. 28.

https://digitalcommons.unl.edu/physicsrudd/28

This Article is brought to you for free and open access by the Research Papers in Physics and Astronomy at DigitalCommons@University of Nebraska - Lincoln. It has been accepted for inclusion in M. Eugene Rudd Publications by an authorized administrator of DigitalCommons@University of Nebraska - Lincoln. 


\section{Authors}

Y.-Y. Hsu, M. W. Gealy, G. W. Kerby III, M. Eugene Rudd, D. R. Schultz, and C. O. Reinhold 


\title{
Energy and angular distributions of electrons from ion impact on atomic and molecular hydrogen. IV. 28-114-keV He ${ }^{+}+\mathrm{H}$ collisions
}

\author{
Y.-Y. Hsu, M. W. Gealy, ${ }^{*}$ G. W. Kerby III, and M. E. Rudd \\ Department of Physics and Astronomy, University of Nebraska, Lincoln, Nebraska 68588-0111 \\ D. R. Schultz and C. O. Reinhold \\ Physics Division, Oak Ridge National Laboratory, Oak Ridge, Tennessee 37831-6373
}

(Received 26 June 1995)

\begin{abstract}
Absolute ionization cross sections for 28-114-keV helium ion impact on atomic hydrogen, differential in energy and angle of the ejected electrons, have been obtained from crossed-beam measurements and previously measured cross sections for molecular hydrogen targets. A radio frequency discharge source produced a mixed atomic and molecular target with a typical dissociation fraction of $74 \%$. Energy spectra were measured from 1.5 to $130 \mathrm{eV}$ by an electrostatic analyzer with a resolution of $5 \%$. The angular range was $15^{\circ}-160^{\circ}$. Results are compared with calculations based on the first Born, continuum-distorted-wave-eikonal-initial-state, and classical trajectory Monte Carlo methods. Total electron yields are obtained by combining calculations that are separately performed for liberating the target and projectile electrons. Model potentials are used to represent the interparticle-separation-dependent screening of the nuclear charges experienced by the electrons. Though projectile electron emission is a negligible component of the total ionization cross section for the present collision energies, its contribution is significant for particular regions of the spectrum of ejected electrons. Through comparison with our proton-impact data [Kerby et al., Phys. Rev. A 51, 2256 (1995)], differences and similarities are demonstrated owing to the common asymptotic charge of these two projectiles but differing nuclear charges and the electron carried by the $\mathrm{He}^{+}$ion. Comparisons are also made illustrating the differences between atomic and molecular hydrogen targets.
\end{abstract}

PACS number(s): 34.50.Fa

\section{INTRODUCTION}

The theoretical description of the ionization of an atom by ion impact can involve many complexities, such as the necessity of treating electron correlation, the ejection of the electron in the combined two-center field of the projectile and target ions, and quasimolecular effects. In order to separate the various effects and to understand the basic mechanisms, experimental data on simple systems (i.e., those containing the fewest electrons) are crucial. In addition, to provide a stringent test of alternative theoretical descriptions of ionization, information more detailed than the total ionization cross section (TICS) is needed, such as that provided by cross sections differential in the angle and energy of ejected electrons. Towards this end, data for the $\mathrm{H}^{+}+\mathrm{H}$ system have been presented in a recent paper [1]. The next level of complexity is the consideration of a collision pair involving an atom and an electron-bearing ion. The simplest system of this type is $\mathrm{He}^{+}+\mathrm{H}$, data for which are presented here.

This work presents measurements of differential cross sections for the ejection of electrons in $\mathrm{He}^{+}+\mathrm{H}$ collisions. Since we make measurements which cover a large angle and energy range, we may also obtain values of the TICS by integration. As far as we know there are no TICS measure-

\footnotetext{
*Present address: Concordia College, Moorhead, MN 56562.
}

ments for this system in the literature. We report these differential and total cross sections for 28, 48, 67, 95, and 114 $\mathrm{keV}$ projectile energies and compare them with theoretical calculations.

The general three-body problem in ion-atom collisions has been treated by various authors [2-5]. However, the $\mathrm{He}^{+}+\mathrm{H}$ combination constitutes a four-body system. Previous theoretical calculations have been made primarily at high impact energy where, unlike the present case, electron removal from the projectile is a significant component even in the TICS. For example, Boyd et al. [6] modified the planewave Born approximation formulation of Bates and Griffing [7] to take account of electron emission by the projectile and Manson and Toburen [8] used the Born approximation to calculate differential cross sections for electron emission from $2-\mathrm{MeV} \mathrm{He}^{+}+\mathrm{He}$ collisions. For low impact energies such as those considered here, the probability of removing the electron from the projectile is much smaller than for removing the less tightly bound target electron. Even so, in particular regions of the angle and energy distribution of the ejected electrons, projectile electron emission plays a significant role.

Also, since the collision velocities involved in this work are rather low (i.e., $v_{\text {projectile }} \lesssim 1$ a.u.) quasimolecular effects may play an important role. Unfortunately, there exists no theoretical description which is presently capable of providing double differential ionization cross sections in this energy range. That is, the theories which provide the most accurate description of low-energy ionization are based on a combined atomic and molecular basis expansion, and conse- 
quently it is difficult to include a large enough basis of functions to project simultaneously the electronic angle and energy in the final state. Therefore, to provide a theoretical context for the present experimental measurements, we must apply methods that have been demonstrated to be applicable at somewhat higher energies ( $v_{\text {projectile }} \gtrsim 1$ a.u.). In particular, we have computed cross sections using the first Born (B1), continuum-distorted-wave-eikonal-initial-state (CDWEIS), and classical trajectory Monte Carlo (CTMC) approximations. The interparticle-separation-dependent screening of the projectile nuclear charge due to the electron present in $\mathrm{He}^{+}$is described by incorporating a model potential to describe this interaction. Similarly, to treat ionization of the projectile, a model potential is used to describe the interaction of the electron present in $\mathrm{He}^{+}$with the impinging hydrogen atom.

By comparing the resulting cross sections for $\mathrm{He}^{+}$impact with those for $\mathrm{H}^{+}$impact, differences in the ejected electron spectrum may be found which result from projectile ionization or from very near collisions in which the target electron experiences a different projectile nuclear charge in the two cases. Also, since the two projectiles have the same ionic or asymptotic charge, portions of the ejected electron spectra will be quite similar. Finally, the direct electron-electron interaction may play a role in the ionization process, but here its effect has only been taken into account theoretically through the screening of the nuclear charges.

This is paper IV of a series of works on doubledifferential ionization cross sections (DDCS's) for fundamental systems. In paper I [9] the apparatus and method were described for obtaining DDCS's for $\mathrm{H}$ from the mixed $\mathrm{H}$ and $\mathrm{H}_{2}$ targets produced by the source. Paper II [1] presented a comparison of our experimental measurements for $\mathrm{H}^{+}+\mathrm{H}$ with theoretical results. The experimental method for determining the present cross sections for $\mathrm{He}^{+}+\mathrm{H}$ utilizes the $\mathrm{H}_{2}$ cross sections which were presented in paper III [10]. The effect of excited helium ions in the projectile beam was also discussed in that work.

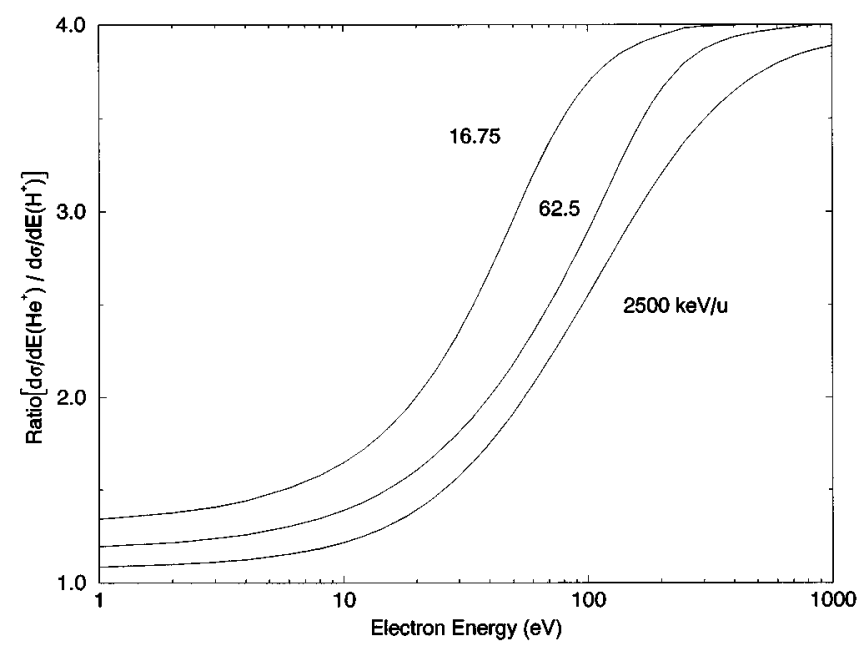

FIG. 1. Ratio of the single-differential ionization cross section for $\mathrm{He}^{+}$impact on atomic hydrogen to that for $\mathrm{H}^{+}$impact, as a function of ejected electron energy, computed in the Born approximation for 16.75-, 62.5-, and 2500-keV/u projectiles for target electron emission.

\section{THEORY}

In this work, we make a comparison of the present experimental measurements with the three most widely applied theoretical methods for ionization in intermediate-energy ion-atom collisions. These methods were described in paper II [1], so we describe only the differences necessary to treat an electron-bearing projectile through a model potential $U_{p}$. To treat projectile electron emission, the roles of target and projectile are reversed and the calculation is repeated. A simple frame transformation then yields the projectile electron spectrum in the laboratory frame, which can be summed with the target electron spectrum to obtain the total electron yield (see Ref. [11] for a more comprehensive description). The role of direct electron-electron interaction is ignored.

The model potential can be easily incorporated within the

TABLE I. Measured values of $\sigma(W, \theta)$ in units of $10^{-20} \mathrm{~cm}^{2} / \mathrm{eV} \mathrm{sr}, \sigma(W)$ in units of $10^{-20} \mathrm{~cm}^{2} / \mathrm{eV}$, $\sigma(\theta)$ in units of $10^{-20} \mathrm{~cm}^{2} / \mathrm{sr}$, and $\sigma_{i}$ (lower right-hand corner) in units of $10^{-20} \mathrm{~cm}^{2}$ for secondary-electron production in $28-\mathrm{keV} \mathrm{He}^{+}+\mathrm{H}$ collisions. Numbers in brackets are powers of 10 by which quantities are to be multiplied.

\begin{tabular}{llllllllll}
\hline \hline$W(\mathrm{eV})$ & \multicolumn{1}{c}{$15^{\circ}$} & \multicolumn{1}{c}{$30^{\circ}$} & \multicolumn{1}{c}{$50^{\circ}$} & \multicolumn{1}{c}{$70^{\circ}$} & $90^{\circ}$ & $110^{\circ}$ & $130^{\circ}$ & $160^{\circ}$ & $\sigma(W)$ \\
\hline 1.5 & 597 & 51.2 & 25.6 & 15.5 & 14.1 & 13.8 & 12.6 & 13.8 & 520 \\
2 & 319 & 41.3 & 26.7 & 12.6 & 11.8 & 10.6 & 10.5 & 12.0 & 352 \\
3 & 82.8 & 36.7 & 18.4 & 9.11 & 8.33 & 6.69 & 7.04 & 9.75 & 185 \\
5 & 67 & 26.1 & 12.7 & 4.79 & 3.69 & 3.15 & 3.55 & 4.20 & 117 \\
7.5 & 38.6 & 17.9 & 6.87 & 2.75 & 1.89 & 1.69 & 1.91 & 2.27 & 67.9 \\
10 & 26.8 & 12.9 & 5.37 & 1.96 & 1.01 & 1.04 & 1.23 & 1.53 & 47.6 \\
15 & 11.1 & 5.40 & 2.32 & 0.640 & 0.556 & 0.534 & 0.586 & 1.10 & 20.8 \\
20 & 4.77 & 2.52 & 0.887 & 0.346 & 0.135 & 0.253 & 0.369 & 0.368 & 9.02 \\
30 & 0.739 & 0.660 & 0.307 & 0.0747 & 0.229 & 0.0497 & 0.0595 & 0.111 & 2.57 \\
50 & 0.129 & 0.113 & 0.186 & 0.0109 & 0.00872 & 0.00415 & 0.00486 & 0.0273 & 0.595 \\
75 & 0.0136 & 0.00769 & 0.00458 & 0.00148 & $4.00[-4]$ & $5.26[-4]$ & $5.60[-4]$ & 0.00976 & 0.0400 \\
100 & $9.19[-4]$ & $8.65[-4]$ & $2.83[-4]$ & $6.86[-4]$ & $2.41[-4]$ & $2.39[-4]$ & $3.16[-4]$ & $9.01[-4]$ & 0.00592 \\
130 & & $1.59[-5]$ & & $2.40[-5]$ & & $1.38[-6]$ & & $3.95[-4]$ & $5.45[-4]$ \\
\hline$\sigma(\theta)$ & 2370 & 389 & 174 & 84.3 & 71.7 & 65.9 & 64.9 & 79.1 & 2530 \\
\hline \hline
\end{tabular}


TABLE II. Same as Table I for $48 \mathrm{keV}$.

\begin{tabular}{|c|c|c|c|c|c|c|c|c|c|}
\hline$W(\mathrm{eV})$ & $15^{\circ}$ & $30^{\circ}$ & $50^{\circ}$ & $70^{\circ}$ & $90^{\circ}$ & $110^{\circ}$ & $130^{\circ}$ & $160^{\circ}$ & $\sigma(W)$ \\
\hline 1.5 & 761 & 181 & 29.8 & 16.7 & 11.5 & 12.6 & 11.5 & 7.82 & 714 \\
\hline 2 & 635 & 116 & 25.8 & 16.6 & 10.3 & 10.9 & 10.4 & 9.66 & 578 \\
\hline 3 & 460 & 69.1 & 22.7 & 13.8 & 8.67 & 10.7 & 9.56 & 9.25 & 430 \\
\hline 5 & 137 & 60.2 & 22.0 & 10.4 & 6.66 & 6.91 & 7.58 & 5.35 & 235 \\
\hline 7.5 & 166 & 60.5 & 18.3 & 7.79 & 4.18 & 4.02 & 4.11 & 3.11 & 216 \\
\hline 10 & 144 & 53.6 & 14.1 & 5.76 & 3.40 & 2.83 & 2.94 & 4.70 & 183 \\
\hline 15 & 78.4 & 33.6 & 8.00 & 2.67 & 1.84 & 1.59 & 2.01 & 1.48 & 103 \\
\hline 20 & 37.0 & 16.8 & 3.86 & 1.36 & 0.879 & 1.19 & 0.871 & 0.947 & 50.7 \\
\hline 30 & 6.72 & 3.75 & 1.06 & 0.365 & 0.485 & 0.236 & 0.282 & 0.313 & 12.0 \\
\hline 50 & 0.289 & 0.305 & 1.19 & 0.0612 & 0.0247 & 0.0230 & 0.0358 & & 2.86 \\
\hline 75 & 0.470 & 0.0441 & 0.0215 & 0.00861 & 0.00248 & 0.00243 & 0.00381 & & 0.347 \\
\hline 100 & 0.00930 & 0.00366 & 0.00369 & 0.00114 & $1.87[-5]$ & $7.05[-4]$ & 0.00947 & & 0.0404 \\
\hline 130 & 0.00130 & 0.00135 & $7.58[-4]$ & $2.64[-4]$ & $1.95[-4]$ & & & & 0.00424 \\
\hline$\sigma(\theta)$ & 4480 & 1410 & 359 & 149 & 101 & 101 & 101 & 83.8 & 5200 \\
\hline
\end{tabular}

first-order Born (B1) approximation since the corresponding $T$ matrix reduces to

$$
T^{\mathrm{B} 1}(\vec{k}, \vec{q})=\tilde{U}_{p}(\vec{q}) \int d^{3} r \psi_{\vec{k}, Z_{t}}^{-*}(\vec{r}) e^{i \vec{q} \cdot \vec{r}} \varphi_{\alpha_{i}}(\vec{r})
$$

where $\varphi_{\alpha_{i}}$ and $\psi_{\vec{k}, Z_{t}}^{-*}$ are the initial and final states of the electron, respectively, $\vec{k}$ is the momentum of the electron, $Z_{t}$ is the target nuclear charge, $\vec{q}$ is the momentum transferred from the projectile, and $\tilde{U}_{p}$ is the Fourier transform of $U_{p}$. Therefore the $T$ matrix for a partially stripped ion with asymptotic charge $Z_{p}$ is related to the $T$ matrix for a fully stripped ion with the same charge by a simple multiplicative factor,

$$
T^{\mathrm{B} 1}=-\left(\frac{q^{2} \tilde{U}_{p}(\vec{p})}{4 \pi Z_{p}}\right) T_{\text {fully stripped }}^{\mathrm{B} 1}
$$

The inclusion of a non-Coulomb projectile field within the CDW-EIS approximation is a much more tedious task which, to our knowledge, has only been achieved for neutral projectiles [12]. We use this procedure to compute the electron emission from $\mathrm{He}^{+}$, in which case the impinging particle is neutral $\mathrm{H}$. Because of its inherent difficulties, the effect of the non-Coulombic projectile core in the CDW-EIS calculation for ionization of $\mathrm{H}$ by $\mathrm{He}^{+}$impact is only estimated here using the same multiplicative factor as in the B1 approximation.

The form chosen for $U_{p}$ was taken from Garvey et al. [13], and is given by a two-parameter formula which fits tabulated Hartree-Fock orbital energies. It is essentially equivalent to the ordinary electrostatic screening potential derived from the charge density given by the wave function of the electron in $\mathrm{He}^{+}$or $\mathrm{H}$. The same potentials have been used to replace the projectile-target-electron interactions in the CTMC calculations.

The basic effect of the model potential used to represent the $\mathrm{He}^{+}$core on the spectrum of electrons ejected is illus-

TABLE III. Same as Table I for $67 \mathrm{keV}$.

\begin{tabular}{llllllllll}
\hline \hline$W(\mathrm{eV})$ & $15^{\circ}$ & $30^{\circ}$ & $50^{\circ}$ & $70^{\circ}$ & $90^{\circ}$ & $110^{\circ}$ & $130^{\circ}$ & $160^{\circ}$ & $\sigma(W)$ \\
\hline 1.5 & 921 & 229 & 39.2 & 23.1 & 8.92 & 8.17 & 6.51 & 8.94 & 844 \\
2 & 787 & 190 & 32.5 & 21.2 & 8.26 & 7.50 & 4.77 & 7.32 & 717 \\
3 & 687 & 137 & 29.9 & 17.3 & 6.94 & 6.08 & 6.16 & 5.25 & 601 \\
5 & 452 & 103 & 29.0 & 13.1 & 6.33 & 5.31 & 5.13 & 3.01 & 436 \\
7.5 & 263 & 102 & 27.5 & 11.7 & 4.74 & 3.84 & 3.70 & 7.47 & 332 \\
10 & 280 & 96.3 & 23.4 & 8.39 & 4.12 & 2.87 & 5.74 & 2.96 & 318 \\
15 & 224 & 77.4 & 15.1 & 4.63 & 2.46 & 4.52 & 1.55 & 1.15 & 237 \\
20 & 140 & 52.9 & 9.55 & 2.49 & 1.59 & 1.47 & 0.893 & 0.891 & 150 \\
30 & 50.2 & 19.1 & 3.15 & 0.935 & 0.980 & 0.403 & 0.388 & 0.368 & 54.4 \\
50 & 3.61 & 1.32 & 2.13 & 0.135 & 0.0639 & 0.0564 & 0.0648 & 7.48 \\
75 & 6.07 & 1.29 & 0.0521 & 0.0193 & 0.00920 & 0.00613 & 0.00919 & 4.38 \\
100 & 0.0549 & 0.0268 & 0.0118 & 0.00323 & 0.00271 & 0.00156 & $8.84[-4]$ & 0.0906 \\
130 & 0.00812 & 0.00606 & 0.00247 & 0.00169 & $1.50[-4]$ & $1.94[-4]$ & $1.70[-4]$ & & 0.0185 \\
\hline$\sigma(\theta)$ & 8860 & 2600 & 585 & 221 & 110 & 98.2 & 91.3 & 76.4 & 9000 \\
\hline \hline
\end{tabular}


TABLE IV. Same as Table I for $95 \mathrm{keV}$.

\begin{tabular}{lllllccccc}
\hline \hline$W(\mathrm{eV})$ & $15^{\circ}$ & $30^{\circ}$ & $50^{\circ}$ & $70^{\circ}$ & $90^{\circ}$ & $110^{\circ}$ & $130^{\circ}$ & $160^{\circ}$ & $\sigma(W)$ \\
\hline 1.5 & 1211 & 457 & 104 & 33.0 & 15.2 & 12.2 & 15.0 & 11.1 & 1380 \\
2 & 1100 & 367 & 85.7 & 29.3 & 11.6 & 11.0 & 11.6 & 10.5 & 1180 \\
3 & 923 & 287 & 67.0 & 21.1 & 9.27 & 9.47 & 8.43 & 9.15 & 953 \\
5 & 720 & 187 & 54.2 & 16.9 & 6.21 & 4.88 & 5.19 & 6.67 & 703 \\
7.5 & 518 & 146 & 46.9 & 14.0 & 4.33 & 3.60 & 4.50 & 3.37 & 534 \\
10 & 377 & 134 & 42.1 & 11.9 & 3.72 & 5.89 & 3.67 & 2.68 & 440 \\
15 & 314 & 119 & 30.3 & 7.07 & 2.59 & 3.00 & 1.49 & 1.49 & 350 \\
20 & 256 & 95.5 & 20.1 & 4.20 & 5.64 & 1.27 & 0.889 & 0.829 & 278 \\
30 & 138 & 54.1 & 8.90 & 2.05 & 0.820 & 0.652 & 0.506 & 0.525 & 143 \\
50 & 21.6 & 8.75 & 1.71 & 0.391 & 0.181 & 0.149 & 0.131 & 0.167 & 23.8 \\
75 & 1.20 & 5.02 & 0.176 & 0.0470 & 0.0184 & 0.0244 & 0.0270 & 0.0717 & 5.94 \\
100 & 0.123 & 0.0926 & 0.0356 & 0.00971 & 0.00343 & 0.00574 & 0.00537 & 0.0233 & 0.288 \\
130 & 0.0178 & 0.0147 & 0.00613 & 0.0237 & $1.99[-4]$ & 0.00108 & $5.60[-4]$ & 0.00564 & 0.0486 \\
\hline$\sigma(\theta)$ & 14000 & 5080 & 1190 & 325 & 146 & 123 & 111 & 99.9 & 15300 \\
\hline \hline
\end{tabular}

trated in Fig. 1. In this case, the Born approximation has been used to compute the ratio of the target single differential cross section (SDCS) as a function of electron energy for $\mathrm{He}^{+}+\mathrm{H}$ to that for $\mathrm{H}^{+}+\mathrm{H}$. The result is plotted for fixed projectile kinetic energy per amu, that is, for equal $\mathrm{He}^{+}$and $\mathrm{H}^{+}$velocities. This figure shows that fast ejected electrons experience the bare nuclear charge of the ion since they originate in close collisions. The $\left[(d \sigma / d E)\left(\mathrm{He}^{+}\right)\right] /$ $\left[(d \sigma / d E)\left(\mathrm{H}^{+}\right)\right]$ratio tends to a value of 4 since the Born approximation scales as the square of the charge. For slow electrons, the ratio is closer to 1 since these electrons originate primarily from distant collisions where the ionic charges of the two projectiles are the same. The deviation from being strictly 1 is due to the fact that even for very close collisions slow electrons can be produced.

We have compared our CTMC total cross sections for target electron emission in $\mathrm{He}^{+}+\mathrm{H}$ with those computed by Willis et al., [14] who used semiempirical potentials and incorporated a more complicated CTMC procedure. The present CTMC TICS's are approximately $25 \%$ smaller than those of Willis et al., and the effects of their approach on the differential cross sections would be difficult to estimate since they were not computed by those authors.

\section{RESULTS}

Detailed descriptions of the apparatus, experimental method, theory of the measurement, and the reliability were given in paper III [10] and will not be repeated here. In addition, much of the physics underlying the spectrum of ejection of electrons in this type of collision was presented and discussed in paper II [1], so we will concentrate here on the new features engendered by the change of projectile. Absolute values of the DDCS's are given in Tables I-V for five incident energies $(28,48,67,95$, and $114 \mathrm{keV})$. The DDCS's were numerically integrated over electron angle or energy to obtain the single differential cross sections (SDCS's) and over both angle and energy to obtain the TICS's, which are also given in the tables.

TABLE V. Same as Table I for $114 \mathrm{keV}$.

\begin{tabular}{llllllllll}
\hline \hline$W(\mathrm{eV})$ & $15^{\circ}$ & $30^{\circ}$ & $50^{\circ}$ & $70^{\circ}$ & $90^{\circ}$ & $110^{\circ}$ & $130^{\circ}$ & $160^{\circ}$ & $\sigma(W)$ \\
\hline 1.5 & 1510 & 647 & 164 & 61.6 & 25.6 & 17.1 & 15.1 & 15.3 & 1910 \\
2 & 1360 & 543 & 140 & 53.6 & 20.4 & 12.6 & 11.7 & 12.8 & 1650 \\
3 & 1090 & 383 & 103 & 41.4 & 16.6 & 10.4 & 7.83 & 16.6 & 1260 \\
5 & 782 & 233 & 68.7 & 25.4 & 8.45 & 5.28 & 8.07 & 6.27 & 831 \\
7.5 & 587 & 178 & 55.7 & 18.9 & 5.28 & 4.53 & 3.58 & 2.78 & 624 \\
10 & 466 & 151 & 47.2 & 14.5 & 4.47 & 3.39 & 1.81 & 2.36 & 507 \\
15 & 341 & 129 & 38.1 & 9.76 & 3.17 & 1.75 & 1.44 & 1.63 & 392 \\
20 & 298 & 102 & 23.7 & 5.51 & 2.44 & 1.11 & 0.722 & 0.766 & 306 \\
30 & 189 & 67.7 & 11.8 & 3.66 & 0.934 & 0.558 & 0.382 & 0.465 & 189 \\
50 & 48.0 & 16.5 & 2.75 & 0.563 & 0.245 & 0.146 & 0.125 & 0.162 & 46.4 \\
75 & 4.49 & 3.93 & 0.592 & 0.0861 & 0.0509 & 0.0319 & 0.0279 & 0.0629 & 7.43 \\
100 & 2.13 & 0.230 & 0.0521 & 0.0151 & 0.0163 & 0.00684 & 0.00569 & 0.0314 & 1.49 \\
130 & 0.0549 & 0.0302 & 0.0131 & 0.00358 & 0.00368 & 0.00141 & 0.00193 & 0.00287 & 0.105 \\
\hline$\sigma(\theta)$ & 17500 & 6370 & 1590 & 517 & 189 & 124 & 106 & 120 & 19500 \\
\hline \hline
\end{tabular}


TABLE VI. Total cross section for ionization in 28-, 48-, 67-, 95-, and 114-keV $\mathrm{He}^{+}$impact of atomic hydrogen. The present measurements of the double-differential cross section have been integrated to yield the total cross section. Also tabulated are the present CTMC, CDW-EIS, and B1 results. Numbers marked with an asterisk give the contribution from projectile electron emission while the other cross sections represent the sum of target and projectile emission. All cross sections here are given in units of $10^{-16} \mathrm{~cm}^{2}$.

\begin{tabular}{|c|c|c|c|c|c|}
\hline \multirow[b]{2}{*}{ Energy } & \multirow[b]{2}{*}{ Energy/u } & \multicolumn{4}{|c|}{ Total ionization cross section } \\
\hline & & Experiment & CTMC & CDW-EIS & B1 \\
\hline \multirow[t]{2}{*}{$28 \mathrm{keV}$} & $7 \mathrm{keV}$ & 0.253 & 0.049 & 0.053 & 1.5 \\
\hline & & & $0.0011 *$ & $0.030 *$ & $0.0031 *$ \\
\hline \multirow[t]{2}{*}{48} & 12 & 0.52 & 0.25 & 0.33 & 2.4 \\
\hline & & & $0.0047^{*}$ & $0.081 *$ & $0.013 *$ \\
\hline \multirow[t]{2}{*}{67} & 16.75 & 0.9 & 0.49 & 0.79 & 2.8 \\
\hline & & & $0.012 *$ & $0.14 *$ & $0.024 *$ \\
\hline \multirow[t]{2}{*}{95} & 23.75 & 1.53 & 0.82 & 1.5 & 3.0 \\
\hline & & & $0.026 *$ & $0.19^{*}$ & $0.040 *$ \\
\hline \multirow[t]{2}{*}{114} & 28.5 & 1.95 & 1.0 & 1.9 & 3.0 \\
\hline & & & $0.042 *$ & $0.22 *$ & $0.048 *$ \\
\hline
\end{tabular}

\section{A. Total cross sections}

A number of interesting features may be seen by inspection of the TICS's for $\mathrm{He}^{+}+\mathrm{H}, \mathrm{H}_{2}$ and by comparing them with TICS's for $\mathrm{H}^{+}+\mathrm{H}, \mathrm{H}_{2}$. To begin with, the present experimental measurements are compared with theoretical results in TableVI. The results of the CTMC and CDW-EIS calculations substantially underestimate the cross section at the lowest impact energies, but the CDW-EIS approximation reaches the level of the experiments at the highest energy measured, while the CTMC method still underestimates the TICS by a factor of almost two. The B1 approximation is significantly larger than experiment for all impact energies
TABLE VII. Ratio of TICSs for $\mathrm{H}^{+}$or $\mathrm{He}^{+}$impact on molecular hydrogen and atomic hydrogen $\left[\sigma\left(\mathrm{H}_{2} / \sigma(\mathrm{H})\right]\right.$ as a function of collision energy per amu. Cross sections are for the present experiments for $\mathrm{He}^{+}+\mathrm{H}, \mathrm{H}_{2}$ [10] and our previous measurements for $\mathrm{H}^{+}+\mathrm{H}, \mathrm{H}_{2}[9,1]$. Also shown are the results of measurements by Shah and Gilbody for $\mathrm{H}$ targets [16] $\left(^{*}\right)$ and $\mathrm{H}_{2}$ targets [15] $\left(^{\dagger}\right)$. All cross sections are in units of $10^{-16} \mathrm{~cm}^{2}$.

\begin{tabular}{|c|c|c|c|c|c|}
\hline \multirow[b]{2}{*}{ Energy/u } & \multicolumn{4}{|c|}{ Total ionization cross section } & \multirow{2}{*}{$\frac{\text { Ratio }}{\left(\sigma\left(\mathrm{H}_{2}\right) / \sigma(\mathrm{H})\right.}$} \\
\hline & $\mathrm{H}^{+}+\mathrm{H}$ & $\mathrm{H}^{+}+\mathrm{H}_{2}$ & $\mathrm{He}^{+}+\mathrm{H}$ & $\mathrm{He}^{+}+\mathrm{H}_{2}$ & \\
\hline $7 \mathrm{keV} / \mathrm{u}$ & & & 0.253 & 0.817 & 3.23 \\
\hline 12 & & & 0.52 & 1.14 & 2.19 \\
\hline 16.75 & & & 0.9 & 1.59 & 1.77 \\
\hline 20 & 0.781 & 1.03 & & & 1.32 \\
\hline 23.75 & & & 1.53 & 1.59 & 1.04 \\
\hline 28.5 & & & 1.95 & 2.63 & 1.35 \\
\hline 45 & $1.399 *$ & $1.98^{\dagger}$ & & & 1.42 \\
\hline 48 & 1.44 & 2.14 & & & 1.49 \\
\hline 67 & 1.63 & 2.39 & & & 1.47 \\
\hline 67 & $1.342 *$ & $2.15^{\dagger}$ & & & 1.60 \\
\hline 95 & 1.48 & 2.41 & & & 1.63 \\
\hline 100 & $1.116 *$ & $2.02^{\dagger}$ & & & 1.81 \\
\hline 114 & 1.17 & 1.97 & & & 1.68 \\
\hline 200 & $0.707 *$ & $1.33^{\dagger}$ & & & 1.88 \\
\hline 500 & $0.388 *$ & $0.728^{\dagger}$ & & & 1.88 \\
\hline 1000 & $0.197 *$ & $0.400^{\dagger}$ & & & 2.03 \\
\hline 1500 & $0.139 *$ & $0.300^{\dagger}$ & & & 2.16 \\
\hline
\end{tabular}

considered. The agreement of experiment and theory improves for increasing impact energy as expected. However, this impact energy range is clearly too low for the bulk of the ionizing events (i.e., low-energy electron production) to be simulated within the assured range of applicability of these theories (see the discussion in paper II [1] of the range of applicability of these theories). They do give insight as to the

TABLE VIII. Ratio of total ionization cross sections for $\mathrm{He}^{+}$impact of $\mathrm{H}$ to that for $\mathrm{H}^{+}+\mathrm{H}$. The experimental ratios have been computed by using the present measurements for $\mathrm{He}^{+}$impact and dividing by values interpolated for $\mathrm{H}^{+}$measured by Pieksma et al. [17] and Shah and Gilbody [16]. Theoretical ratios have been directly computed from calculations for each projectile, neglecting projectile ionization. Also shown are experimental ratios for $\mathrm{He}^{+}$and $\mathrm{H}^{+}$impact of $\mathrm{H}_{2}$. In this case, the $\mathrm{He}^{+}$data are from the present measurements and the $\mathrm{H}^{+}$impact cross sections are from values recommended on the basis of a number of existing experiments and theories [18]. The experimental ratio at $500 \mathrm{keV} / \mathrm{u}$ was taken from these recommended data as well.

\begin{tabular}{|c|c|c|c|c|c|}
\hline \multirow[b]{2}{*}{ Energy/u } & \multirow{2}{*}{$\frac{\sigma\left(\mathrm{He}^{+}+\mathrm{H}_{2}\right) / \sigma\left(\mathrm{H}^{+}+\mathrm{H}_{2}\right)}{\text { Experiment }\left(\mathrm{H}_{2}\right)}$} & \multicolumn{4}{|c|}{$\sigma\left(\mathrm{He}^{+}+\mathrm{H}\right) / \sigma\left(\mathrm{H}^{+}+\mathrm{H}\right)$} \\
\hline & & Experiment $(\mathrm{H})$ & СТMC & CDW-EIS & B1 \\
\hline $7 \mathrm{keV}$ & 4.59 & 2.34 & 1.72 & 1.89 & 1.84 \\
\hline 12 & 3.49 & 1.92 & 1.56 & 1.73 & 1.68 \\
\hline 16.75 & 3.22 & 1.81 & 1.85 & 1.61 & 1.62 \\
\hline 23.75 & 1.81 & 1.76 & 1.66 & 1.64 & 1.58 \\
\hline 28.5 & 2.19 & 1.76 & 1.49 & 1.63 & 1.56 \\
\hline 62.5 & & & 1.19 & 1.59 & 1.53 \\
\hline 500 & 1.81 & & 1.76 & 1.43 & 1.42 \\
\hline 2500 & & & 2.53 & 1.35 & 1.39 \\
\hline 25000 & & & 2.84 & 1.28 & 1.32 \\
\hline 250000 & & & 3.79 & 1.23 & 1.27 \\
\hline
\end{tabular}



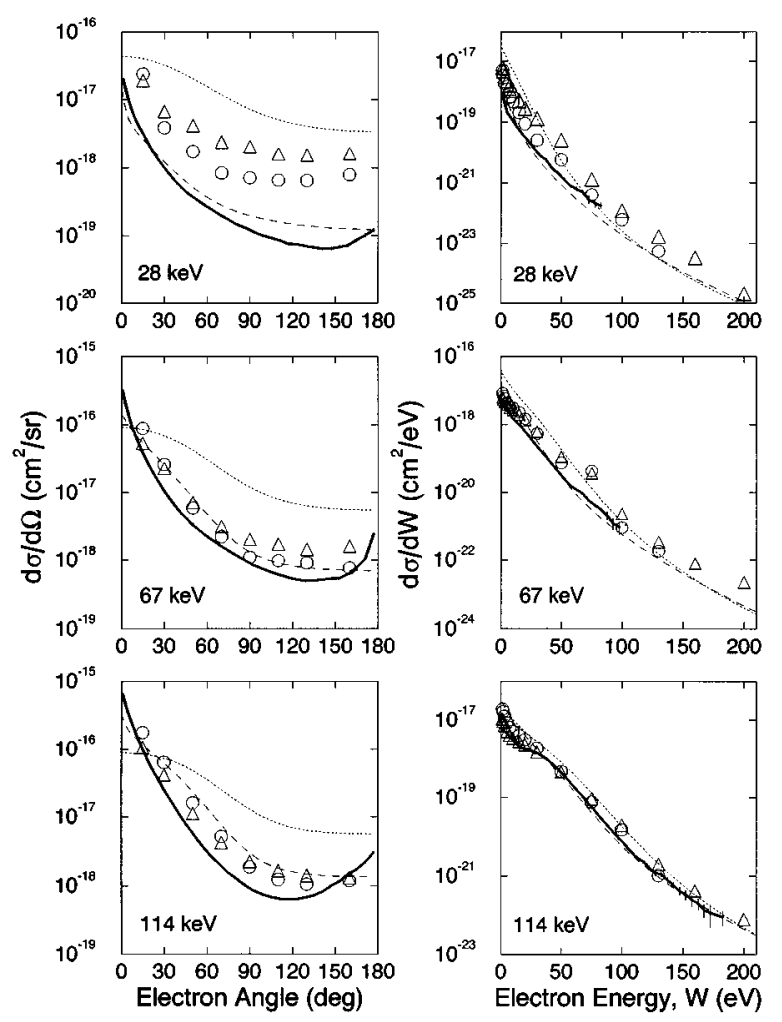

FIG. 2. Single-differential cross section for ionization as a function of electron ejection angle and energy for 28-, 67-, and 114-keV $\mathrm{He}^{+}$impact on $\mathrm{H}$ and $\mathrm{H}_{2}$. Circles, present experiment for $\mathrm{H}$ targets; triangles, present experiment [10] for $\mathrm{H}_{2}$ targets divided by 2; solid line, CTMC; dashed line, CDW-EIS; dotted line, Born approximation. All theoretical calculations are for atomic hydrogen targets and include electron emission from both the target and projectile.

relative contributions of projectile and target electron emission, and the role of screening of the nuclear charges on the spectrum of ejected electrons.

The measured data for impact on either atomic or molecular hydrogen, along with other data available in the literature, help illustrate the differences in the total yield of electrons between these two targets (see Table VII). A simple picture might suggest that, since the binding energies and wave functions are quite similar in $\mathrm{H}$ and $\mathrm{H}_{2}$, twice as many electrons would be ionized from $\mathrm{H}_{2}$ as from $\mathrm{H}$. This simple model is valid at high impact energies when the dominant electron removal channel is direct single ionization. For lower energies, the behavior of this ratio is governed by the competition among different electron removal channels including the two-electron processes possible for $\mathrm{H}_{2}$ (double ionization, transfer ionization, and dissociative ionization). For example, the present results at the lowest impact energies show that the ratio can be significantly larger than 2 in this limit. Then, as impact energy is increased, the ratio reaches a minimum near 1, a result that Shah and Gilbody [15] attributed to the contribution of transfer ionization. That is, in transfer ionization, fewer electrons are liberated to the continuum on average and the ratio is therefore less than 2.

The present experiment and theory allow comparison not only of the differences between $\mathrm{H}$ and $\mathrm{H}_{2}$ targets, but also differences arising from the variation of projectile nuclear

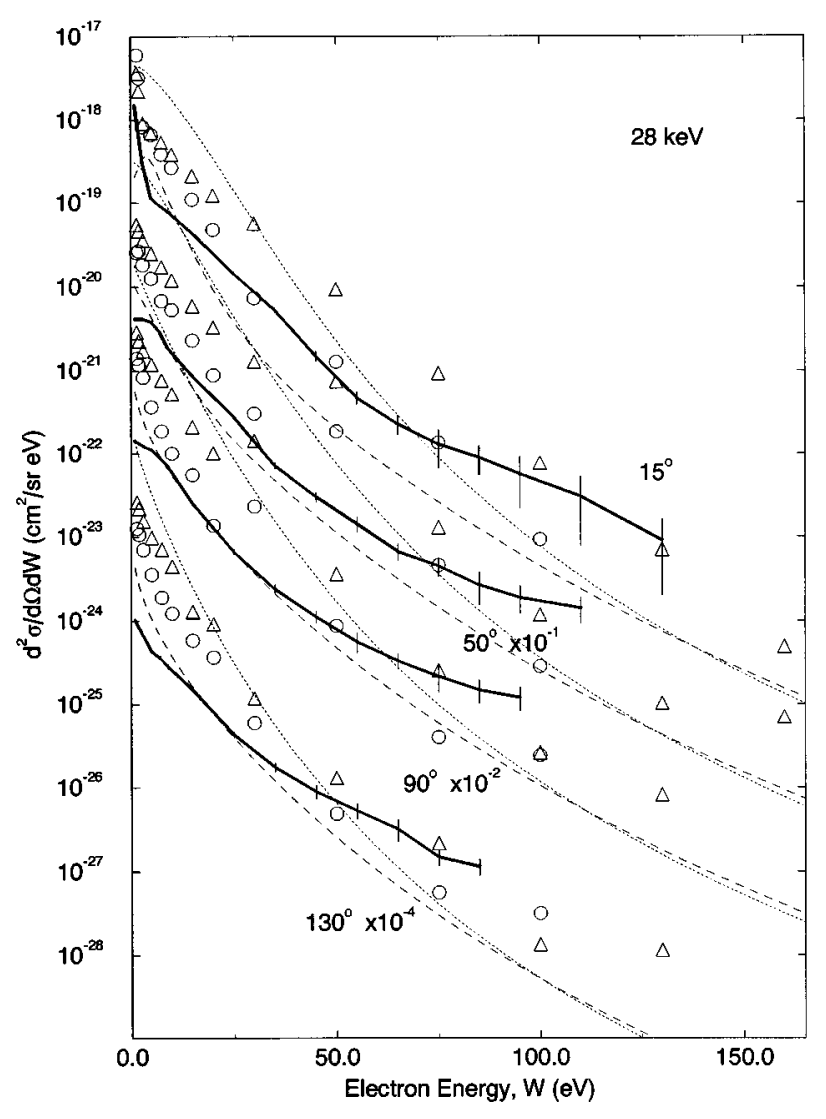

FIG. 3. Double-differential cross section for ionization as a function of ejection energy for $28-\mathrm{keV} \mathrm{He}^{+}$impact on $\mathrm{H}$ and $\mathrm{H}_{2}$, at a wide range of ejection angles $\left(15^{\circ}, 50^{\circ}, 90^{\circ}\right.$, and $\left.130^{\circ}\right)$. The symbols are as indicated in Fig. 2.

charge for a fixed ionic charge. Table VIII gives the ratio of the TICS for $\mathrm{He}^{+}$impact divided by that for $\mathrm{H}^{+}$impact at equal velocities. Perhaps the most obvious conclusion one may draw is that this ratio is consistently greater than 1 , implying that there is always a significant contribution from close collisions between the target electron and the projectile nucleus. In the absence of this penetration of the projectile core, the ratio would be 1 . This penetration affects not only one-electron processes but also two-electron processes, as evidenced by the ratio displayed for $\mathrm{H}_{2}$ targets. In this case, the enhancement of the electron production for $\mathrm{He}^{+}$impact over that for $\mathrm{H}^{+}$impact is even larger than for $\mathrm{H}$ targets. A small contribution also comes from the ejection of electrons from $\mathrm{He}^{+}$, but at these impact energies theory predicts that this accounts for only a few percent of the TICS.

Regarding the experimental measurements, the largest enhancement due to the electron carried by the $\mathrm{He}^{+}$projectile occurs for the lowest collision velocities. The theoretical calculations also show this trend except that the ratio for the CTMC calculation also begins to rise at the highest energies. Quantum mechanically, dipole-allowed large impact parameter collisions play a dominant role at the higher collision energies, and therefore the ratio is smaller, $\mathrm{H}^{+}$and $\mathrm{He}^{+}$ looking more similiar. However, dipole transitions are classically suppressed and only small impact parameter collisions are effective in removing an electron. Therefore the $\mathrm{He}^{+}$ion with its larger nuclear charge produces more ionization and the ratio eventually increases with increasing energy 


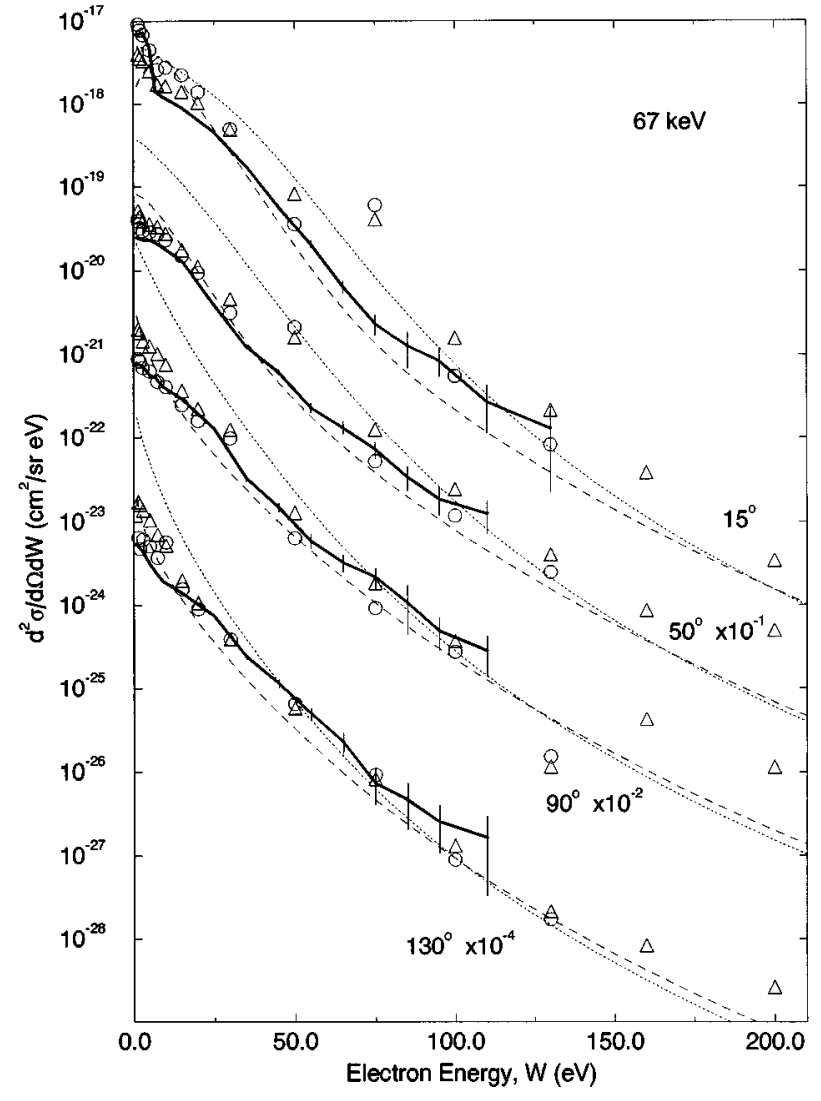

FIG. 4. Same as Fig. 3 except for $67-\mathrm{keV} \mathrm{He}^{+}$impact.

for the CTMC model. Moreover, the classical enhancement of the ratio is associated with the correlation between the position and momentum of the electron violating the Heisenberg uncertainty principle, which makes the ionization probability diverge at small impact parameters [19].

\section{B. Single-differential cross sections}

In Fig. 2, we illustrate the behavior of the singledifferential cross sections for three impact energies spanning the present measurements. For the highest energy displayed, $114 \mathrm{keV}$, the ratio of the projectile velocity to that of the target electron in its initially bound state, $v_{\text {projectile }} / v_{\text {electron }}=1.07$, is high enough so that the range of validity of the CTMC and CDW-EIS approximations is reached, but only at the lower limit [1]. For example, at this energy reasonable agreement is observed among all the theoretical approaches, and with the experimental measurements, regarding the energy distribution of the ejected electrons. This spectrum shows that the emission of electrons is dominated by ejection of low-energy electrons. A shoulder or plateau is observed at around $45 \mathrm{eV}$ which is due to the binary encounter peak in the DDCS, summed over all angles of ejection. Beyond this region the cross section drops off rapidly. Compared to proton impact at the same velocity, this shoulder is more pronounced due to the penetration of the electron into the $\mathrm{He}^{+}$core. This screening enhances the fast electron yield relative to the slow electron yield as demonstrated in Fig. 1.

The agreement between theories and experiment for 114 $\mathrm{keV}$ is not as complete regarding the angular distribution of

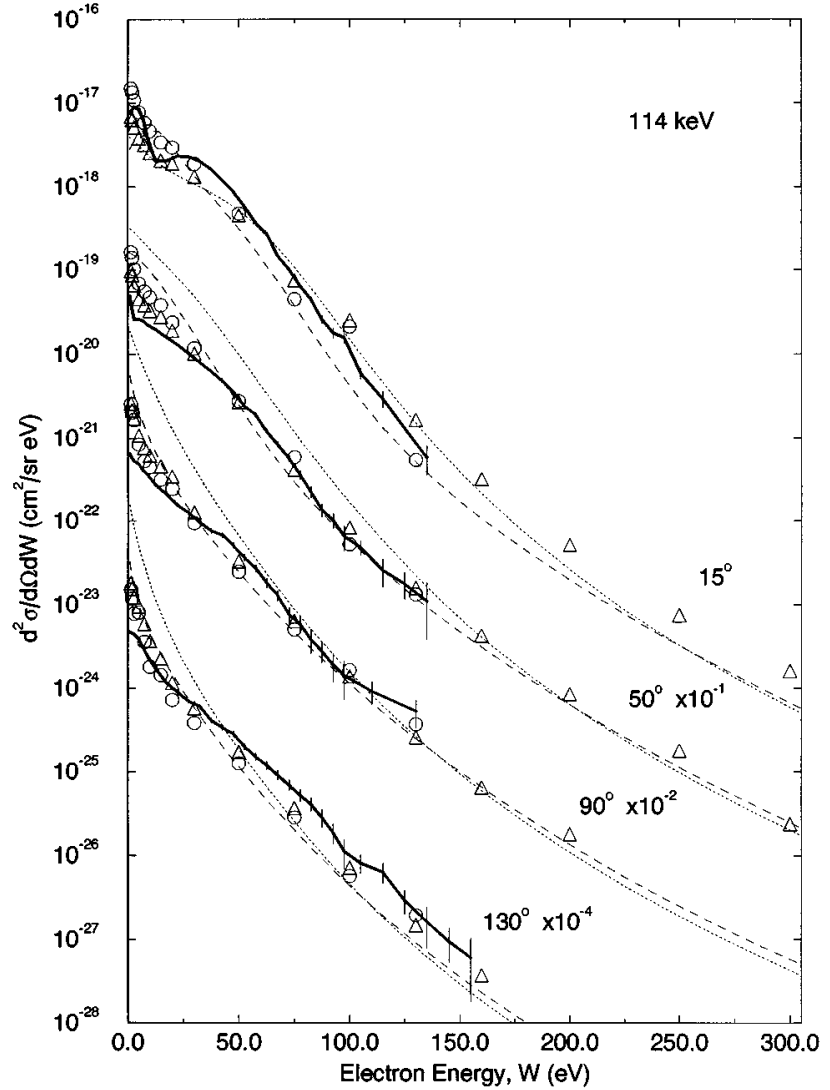

FIG. 5. Same as Fig. 3 except for $114-\mathrm{keV} \mathrm{He}^{+}$impact.

electrons and a well known failure of the B1 approximation is seen at small angles. In this portion of the spectrum, it is critical to represent the outgoing electron as evolving in the combined field of both the projectile and residual target ions since production of "saddle-point" and "cusp" electrons play a very important role. The CDW-EIS and CTMC approximations account for these interactions and give a better agreement with experiment, predicting reasonably well the shape of the angular SDCS.

The largest contribution to the SDCS from projectile electron emission is present for the CDW-EIS model, increasing significantly the fast and backward electron ejection. At an impact energy of $114 \mathrm{keV}$, without the projectile electron emission CDW-EIS underestimates the experimental SDCS by one order of magnitude at $100 \mathrm{eV}$ electron energy and by two orders of magnitude at $200 \mathrm{eV}$ or for angles greater than about $100^{\circ}$. The most notable effect on the CTMC cross section from projectile electron emission results in an upturn of the SDCS for large ejection angles. For the B1 approximation, the contribution is small except for the highest electron energies depicted. In addition, the theories do a progressively worse job of reproducing well the angular distribution of ejected electrons for the lower collision energies, but yield reasonable agreement with the experiment regarding the distribution of ejected electron energies.

\section{Double-differential cross sections}

Displayed in Figs. 3 through 5 are the double-differential ionization cross sections for 28,67 , and $114 \mathrm{keV} \mathrm{He}^{+}$impact of $\mathrm{H}$ and $\mathrm{H}_{2}$. Just as the SDCS represents a more stringent test of theory and experiment than does a comparison of 


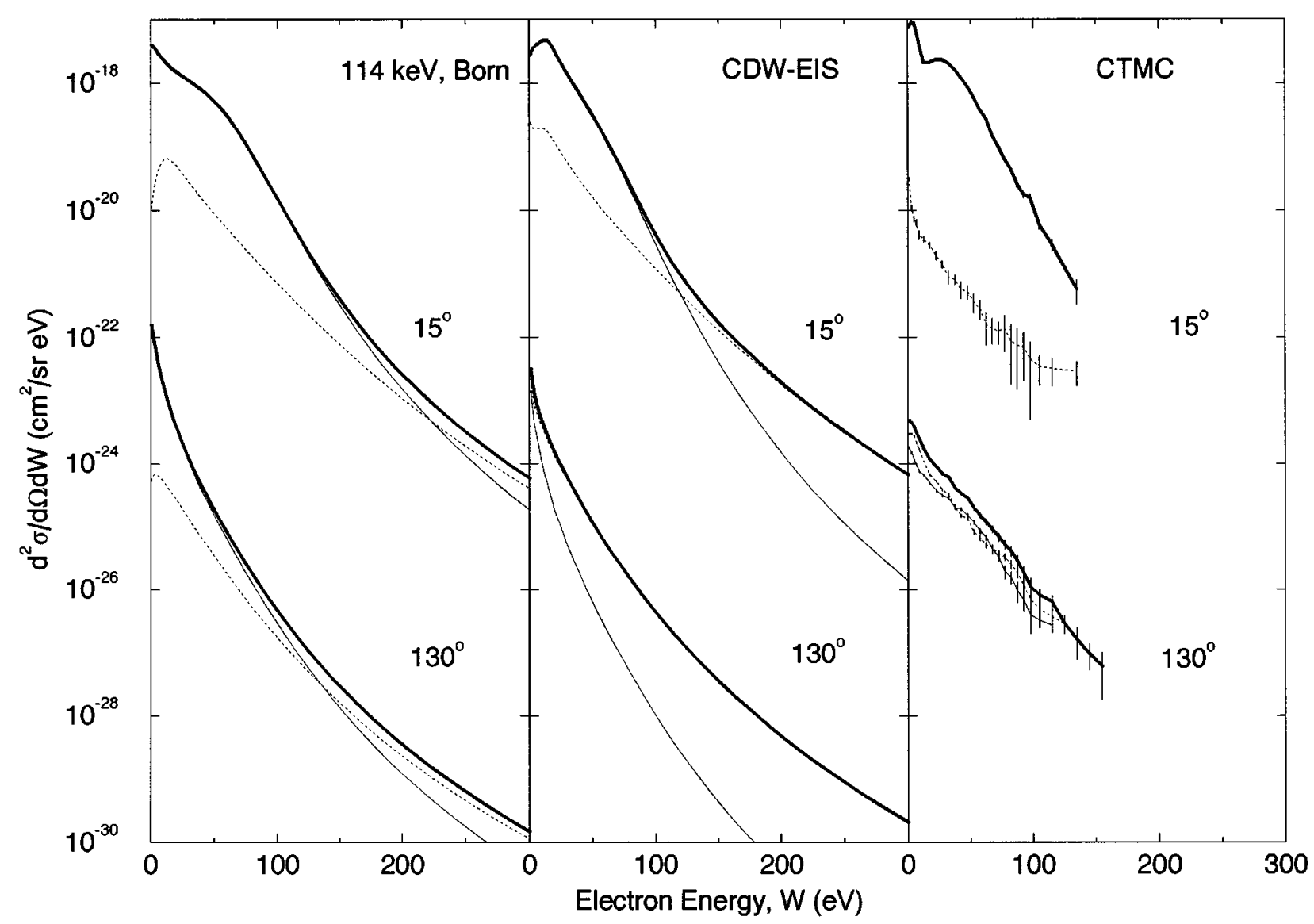

FIG. 6. Illustrated here is the contribution to the DDCS (solid line) from electron emission from the target (light solid line) and from the projectile (dashed line) for $114-\mathrm{keV} \mathrm{He}^{+}$impact on $\mathrm{H}$ in the first Born, CDW-EIS, and CTMC approximations.

only total cross sections, the DDCS likewise provides a more detailed discriminant than the SDCS.

For $28 \mathrm{keV}$, there does not appear to be good agreement between any of the theories and the experiment, but for the higher impact energies at least reasonable agreement begins to be obtained. Perhaps surprisingly, the B1 approximation reproduces the intermediate and fast electrons fairly well, but significantly overestimates the slow electron yield. The CTMC results seem to describe satisfactorily the fast electron cross sections, but underestimate the slow electron production. At low electron energies both the CTMC calculation and the experiments display a rapid change of slope. This is due to a remainder of the electron-capture-to-the-continuum cusp at larger ejection angles [9].

The good general agreement of the CDW-EIS results with the measurements is found to be fortuitous in that the target electron emission is expected to be substantially underestimated (as demonstrated in paper II [1]) and the projectile electron emission appears to be significantly overestimated. The specific contribution from target and projectile electron emission is illustrated in Fig. 6 for each of the theories as a function of electron energy for both a small and large ejection angle. One immediately sees that projectile electron emission is most significant at backward angles. This is because most electrons removed from the projectile are ejected with low energy and are emitted into the forward direction in the projectile frame, which is the backward direction in the target frame. This is simply the normal behavior of the spectrum of electrons ejected in ion-atom collisions, transformed into the laboratory frame. For example, note that the target electron contribution is dominant at small energies for forward emission. Also note that projectile electron emission becomes dominant for high ejection energies because the $\mathrm{He}^{+}$electron has a greater binding energy and therefore a broader electronic momentum distribution. The relative contributions of target and projectile electron emission, and the crossover point in ejection energy where projectile electron emission becomes dominant, are quite different for the three theories. Based on our experience and the known limits of validity of these approximations, we expect the behavior of the CTMC results to be closest to that which occurs in actuality.

In order to directly compare $\mathrm{H}^{+}$and $\mathrm{He}^{+}$impact at equal velocities, in Fig. 7 we display our previous results for 20 $\mathrm{keV}$ ( $v_{\text {projectile }}=0.895$ a.u.) $\mathrm{H}^{+}+\mathrm{H}, \mathrm{H}_{2}$ and the present measurements for $67 \mathrm{keV}\left(v_{\text {projectile }}=0.819\right) \mathrm{He}^{+}+\mathrm{H}$, $\mathrm{H}_{2}$. The most striking feature of this comparison is the enhanced production of hot electrons in the case of $\mathrm{He}^{+}$impact due to the greater nuclear charge and projectile electron emission. The slow electron production, resulting mostly from distant collisions, is more nearly the same for the two projectiles of equal asymptotic charge. This enhancement is very clear, say for $15^{\circ}$ ejection, in the increased magnitude 


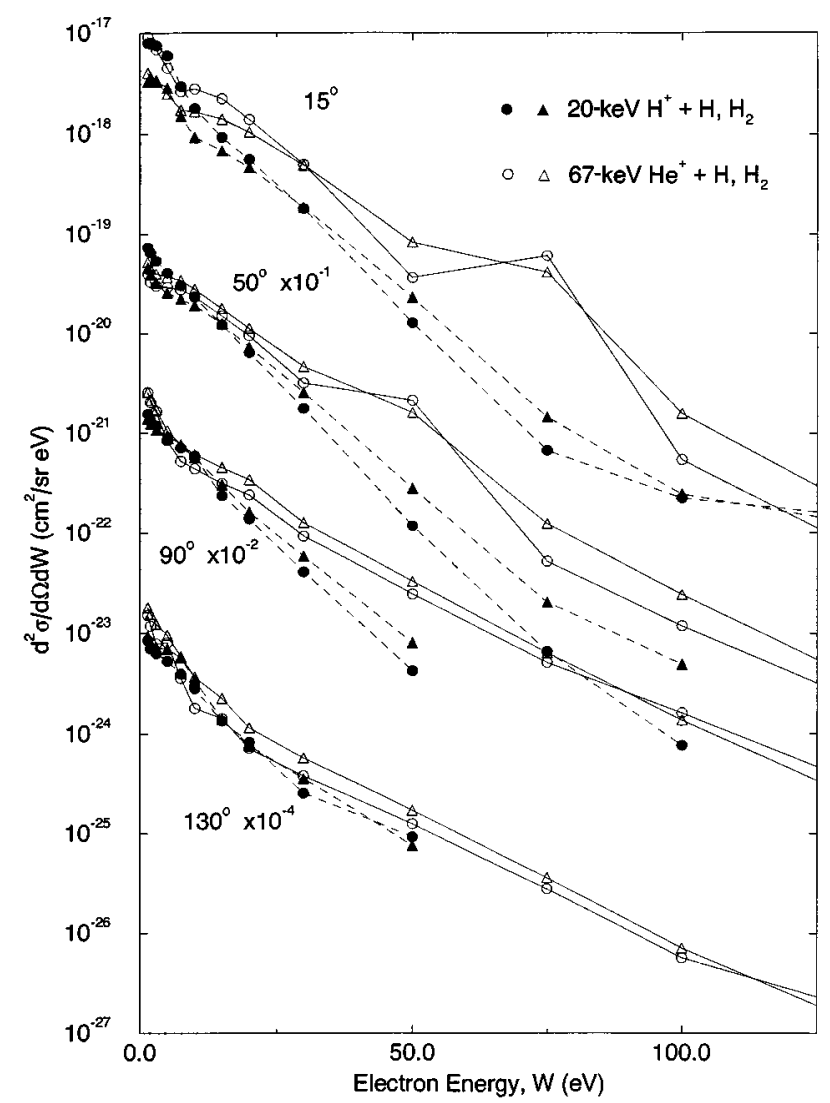

FIG. 7. Comparison of near equivelocity $\mathrm{H}^{+}$and $\mathrm{He}^{+}$impact of $\mathrm{H}$ and $\mathrm{H}_{2}$. $\left(20-\mathrm{keV} \mathrm{H}^{+}\right.$corresponds to a velocity of 0.895 a.u. while $67-\mathrm{keV} \mathrm{He}^{+}$corresponds to a velocity of 0.819 a.u.) Solid symbols, $\mathrm{H}^{+}$impact; open symbols, $\mathrm{He}^{+}$impact; circles, present experiment for $\mathrm{H}$ targets $\left(\mathrm{H}^{+}+\mathrm{H}[1]\right)$; triangles, present experiment for $\mathrm{H}_{2}$ targets divided by $2\left(\mathrm{H}^{+}+\mathrm{H}_{2}[9]\right)$.

of the binary peak. Also evident in this figure are Dopplershifted projectile autoionization peaks (e.g., for $15^{\circ}$ at about $75 \mathrm{eV}$, or for $130^{\circ}$ at about $12 \mathrm{eV}$ ) for the $\mathrm{He}^{+}$data, not present for $\mathrm{H}^{+}$impact.

\section{CONCLUSIONS}

In this work we have reported measurements of the double-differential ionization cross sections for $28-114-\mathrm{keV}$ $\mathrm{He}^{+}+\mathrm{H}$, along with single-differential and total cross sections obtained by integration. We have also compared these data to different theoretical approaches to the calculation of the ejected electron spectrum. Since this represents the simplest collision system involving a partially stripped projectile, it provides a very fundamental test. The primary difference found in this work is that, relative to proton impact, $\mathrm{He}^{+}$produces a greater yield of fast electrons, as would be expected. We have also drawn conclusions regarding the difference between $\mathrm{H}$ and $\mathrm{H}_{2}$ targets.

Since close-coupling treatments are still impractical for the computation of DDCSs, we have utilized the three most commonly applied approaches, the first Born, CDW-EIS, and CTMC approximations, modified to include the screening of the target and projectile nuclei through use of model potentials. Especially at the lowest energy considered, it is clear that fuller treatments of ionization must be developed, especially for low-energy electron emission. For higher impact energies, the CTMC and CDW-EIS approaches provide a reasonable description of the ejected electron spectra, accounting in particular for two-center effects. However, the good general agreement of the CDW-EIS results with the present measurements is attributable to a fortuitous summing of its significant overestimation of projectile electron emission and underestimation of target electron emission.

\section{ACKNOWLEDGMENTS}

The experimental work was supported by the National Science Foundation under Grant Nos. PHY9020529 and PHY9119818. Support of the theoretical work has been provided by the U.S. Department of Energy, Office of Fusion Energy and Office of Basic Energy Sciences through Contract No. DE-AC05-84OR21400 with Oak Ridge National Laboratory, managed by Lockheed Martin Energy Systems, Inc.
[1] G.W. Kerby III, M.W. Gealy, Y.-Y. Hsu, M.E. Rudd, D.R. Schultz, and C.O. Reinhold, Phys. Rev. A 51, 2256 (1995) (paper II).

[2] L.D. Fadeev, Zh. Éksp. Teor. Fiz. 39, 1459 (1960) [Sov. Phys. JETP 12, 1014 (1961)].

[3] L.R. Dodd and K.R. Greider, Phys. Rev. 146, 675 (1966).

[4] Dž. Belkić, J. Phys. B 11, 3529 (1978).

[5] R.E. Olson and A. Salop, Phys. Rev. A 16, 531 (1977).

[6] T.J.M. Boyd, B.L. Moiseiwitsch, and A.L. Stewart, Proc. Phys. Soc. London Sect. A 70, 110 (1957).

[7] D.R. Bates and G. Griffing, Proc. Phys. Soc. London Sect. A 66, 961 (1953).

[8] S.T. Manson and L.H. Toburen, Phys. Rev. Lett. 46, 529 (1981).

[9] M.W. Gealy, G.W. Kerby III, Y.-Y. Hsu, and M.E. Rudd, Phys. Rev. A 51, 2247 (1995) (paper I).

[10] Y.-Y. Hsu, M.W. Gealy, G.W. Kerby III, and M.E. Rudd, pre- ceding paper, Phys. Rev. A 53, 297 (1996).

[11] L.H. Toburen, R.D. DuBois, C.O. Reinhold, D.R. Schultz, and R.E. Olson, Phys. Rev. A 42, 5338 (1990).

[12] J. Wang, C.O. Reinhold, and J. Burgdörfer, Phys. Rev. A 45, 4507 (1992).

[13] R.H. Garvey, C.H. Jackman, and A.E.S. Green, Phys. Rev. A 12, 1144 (1975).

[14] S.L. Willis, G. Peach, M.R.C. McDowell, and J. Banerji, J. Phys. B 18, 3939 (1985).

[15] M.B. Shah and H.B. Gilbody, J. Phys. B 15, 3441 (1982).

[16] M.B. Shah and H.B. Gilbody, J. Phys. B 14, 2361 (1981).

[17] M. Pieksma, S.Y. Ovchinnikov, J. van Eck, W.B. Westerveld, and A. Niehaus, Phys. Rev. Lett. 73, 46 (1994).

[18] C.F. Barnett, Oak Ridge National Laboratory ORNL-6086/V1, 1990 (unpublished).

[19] C.O. Reinhold and J. Burgdörfer, J. Phys. B 26, 3101 (1993). 